EDITORA

OMNIS SCIENTIA

\title{
VOLUME 1
}

\section{Organizadora:}

Havena Mariana dos Santos Souza

\section{ATUAÇÕES DOS}

\section{PROFISSIONAIS}

DE SAÚDE

DURANTE A

\section{RESIDÊNCIA}


EDITORA

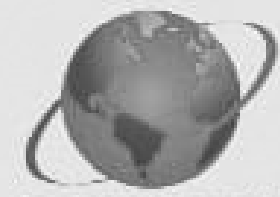

OMNIS SCIENTIA

\section{VOLUME 1}

\section{Organizadora:}

Havena Mariana dos Santos Souza

ATUAÇÕES DOS

\section{PROFISSIONAIS}

DE SAÚDE

DURANTE A

RESIDÊNCIA 
Editora Omnis Scientia

ATUAÇÕES DOS PROFISSIONAIS DE SAÚDE DURANTE A RESIDÊNCIA

Volume 1

$1^{\mathrm{a}}$ Edição

TRIUNFO - PE 


\section{Editor-Chefe}

Me. Daniel Luís Viana Cruz

Organizador (a)

Me. Havena Mariana dos Santos Souza

\section{Conselho Editorial}

Dra. Pauliana Valéria Machado Galvão

Dr. Wendel José Teles Pontes

Dr. Walter Santos Evangelista Júnior

Dr. Cássio Brancaleone

Dr. Plínio Pereira Gomes Júnior

Editores de Área - Ciências da Saúde

Dra. Camyla Rocha de Carvalho Guedine

Dra. Cristieli Sérgio de Menezes Oliveira

Dr. Leandro dos Santos

Dr. Hugo Barbosa do Nascimento

Dr. Marcio Luiz Lima Taga

Dra. Pauliana Valéria Machado Galvão

\section{Assistentes Editoriais}

Thialla Larangeira Amorim

Andrea Telino Gomes

Imagem de Capa

Freepik

\section{Edição de Arte}

Vileide Vitória Laranjeira Amorim

\section{Revisão}

Os autores

\section{(cc) $(1) \subseteq$}

Este trabalho está licenciado com uma Licença Creative Commons - Atribuição-

NãoComercial-SemDerivações 4.0 Internacional.

O conteúdo abordado nos artigos, seus dados em sua forma, correção e confiabilidade são de responsabilidade exclusiva dos autores. 


\title{
Dados Internacionais de Catalogação na Publicação (CIP) (eDOC BRASIL, Belo Horizonte/MG)
}

A885 Atuações dos profissionais de saúde durante a residência [livro eletrônico] / Organizadora Havena Mariana dos Santos Souza. Triunfo, PE: Omnis Scientia, 2021.

114 p. : il.

Formato: PDF

Requisitos de sistema: Adobe Acrobat Reader

Modo de acesso: World Wide Web

Inclui bibliografia

ISBN 978-65-88958-44-5

DOI 10.47094/978-65-88958-44-5

1. Saúde pública - Brasil. 2. Sistema Único de Saúde.

3.Residentes (Medicina) - Avaliação. I. Souza, Havena Mariana dos Santos.

CDD 610.737

Elaborado por Maurício Amormino Júnior - CRB6/2422

\author{
Editora Omnis Scientia \\ Triunfo - Pernambuco - Brasil \\ Telefone: +55 (87) 99656-3565 \\ editoraomnisscientia.com.br \\ contato@editoraomnisscientia.com.br
}




\section{PREFÁCIO}

As mudanças na formação dos profissionais de saúde têm ganhado relevância no mundo. No Brasil, o Ministério da Saúde através das Residências Médicas e Multiprofissionais, assume a responsabilidade de orientar a formação desses profissionais para atender às necessidades do Sistema Único de Saúde (SUS).

No entanto, no início da década de 70 apenas o modelo de Pós-graduação na modalidade de Residência Médica era regulamentado, e somente ao final da mesma década outras categoriais profissionais foram incluídas com objetivo de estabelecer um modelo de atenção integral e no desenvolvimento do processo de trabalho integrado entre os profissionais da saúde. De início os programas eram fortemente atrelados à Estratégia de Saúde da Família (ESF), mas atualmente ganham espaço também nos serviços hospitalares.

No contexto atual de pandemia causado pelo SARS-CoV-2, vale destacar a contribuição dos profissionais de saúde residentes prestando a assistência necessária para reduzir a disseminação do vírus, recuperar o estado de saúde dos pacientes, e consequentemente, evitar que os sistemas de saúde ficassem sobrecarregados.

Em nossos livros selecionamos um dos capítulos para premiação como forma de incentivo para os autores, e entre os excelentes trabalhos selecionados para compor este livro, o premiado foi o capítulo 7, intitulado "TELESSAÚDE COMO FERRAMENTA PARA CAPACITAÇÃO PROFISSIONAL DURANTE A PANDEMIA DO SARS-COV-2". 


\section{SUMÁRIO}

CAPÍTULO 1.

ATUAÇÃO DE RESIDENTES EM SAÚdE DA FAMÍLIA DURANTE A PANDEMIA DE COVID-19

Danieli Cristina Scalco

Eli Fatima Monauer

Luana Lunardi Alban

Maria José Alves Boa Sorte Rodrigues

Matheus Henrique Rossatto

DOI: 10.47094/978-65-88958-44-5/13-18

CAPÍTULO 2.

FORMAÇÃO DOS RESIDENTES MÉDICOS E OS IMPACTOS GERADOS PELA PANDEMIA DO COVID-19

Hemerson Garcia de Oliveira Silva

Thays Carvalho Caldeira Coelho

Marcos Vinícius Mendes Barroso

Amanda Batista Martins Silva

Henrique de Souza Rodrigues Fajardo

Thaís Ker Bretas Werner

Izabella Silva Figueiredo

Lucianne Vanelle Sales Freitas

Fernanda Caldeira Ferraz Batista

Poliana Gomes Pereira

DOI: 10.47094/978-65-88958-44-5/19-26 
O COMPROMISSO BIOÉTICO DA ENFERMAGEM, FRENTE A VACINAÇÃO CONTRA COVID-19 NO ESTADO DO AMAPÁ

Alana Corrêa Santos

Sarah Bianca Trindade

Luiza Soares Pinheiro

Márcia Eduarda Dias Conceição

Vanessa Gomes de Souza

Camila Rodrigues Barbosa Nemer

Luzilena de Sousa Prudêncio

Nely Dayse Santos da Mata

DOI: 10.47094/978-65-88958-44-5/27-34

CAPÍTULO 4 35

UM NOVO OLHAR DO PROFISSIONAL DE EDUCAÇÃO FÍSICA RESIDENTE NA SAÚDE MENTAL: RELATO DE EXPERIÊNCIA

Maria de Lourdes Lima Ferreira

Namir da Guia

DOI: 10.47094/978-65-88958-44-5/35-42

CAPÍTULO 5 43

RELEVÂNCIA DA INTERVENÇÃO DOS RESIDENTES DE EDUCAÇÃO FÍSICA NA SALA DE ESPERA EM TEMPOS PÂNDEMICOS: RELATO DE EXPERIÊNCIA

Camilla Ytala Pinheiro Fernandes

Ágna Retyelly Sampaio de Souza

Lis Maria Machado Ribeiro Bezerra

DOI: $10.47094 / 978-65-88958-44-5 / 43-50$ 
O USO DE TECNOLOGIA DA INFORMAÇÃO PARA O ENFRENTAMENTO À PANDEMIA DA COVID-19 EM CASCAVEL/PR

Felipe Gustavo de Bastiani

Matheus Chaves Veronezzi

Evelyn Farias

Yasmin Luisa Dengo Lombardo

Rafaela Zulmira de Oliveira Moraes

Caroline Solana de Oliveira

Mariana Carvalho de Olivera

Gilson Fernandes da Silva

Paulo Guilherme Bittencourt Marchi

Luis Henrique Cerqueira Vila Verde

DOI: 10.47094/978-65-88958-44-5/51-62

CAPÍTULO 7. .63

TELESSAÚDE COMO FERRAMENTAPARA CAPACITAÇÃO PROFISSIONALDURANTE A PANDEMIA DO SARS-COV-2

Natalya Juliana da Silva

Maria Eduarda de Araújo Nogueira

Maria Gabriella Leite Silva

Amanda Soares de Vasconcelos

Amanda de Figueirôa Silva

José Reinaldo Madeiro Junior

Carolina Albuquerque da Paz

Nara Miranda Portela

DOI: $10.47094 / 978-65-88958-44-5 / 63-70$ 
USO DO 'SOCRATIVE' COMO FERRAMENTA DE ENSINO NA ÁREA DA SAÚDE: REVISÃO INTEGRATIVA

Larissa Artimos Ribeiro

Amanda Curiel Trentin Corral

Gisella de Carvalho Queluci

DOI: $10.47094 / 978-65-88958-44-5 / 71-78$

CAPÍTULO 9.

A IMPORTÂNCIA DA TERRITORIALIZAÇÃO PARA ATUAÇÃO COMO RESIDENTE EM SAÚDE DA FAMÍLIA

Morgana Gomes Izidório

Pedro Ítalo Alves de Carvalho

Breno Carvalho de Farias

Jessica Cristina Moraes de Araújo

Maria do Socorro Teixeira de Sousa

Francisco Natanael Lopes Ribeiro

Maria Cassiana Rosa Carneiro Cunha

Luís Fernando Cavalcante do Nascimento

Thaís Fontenele de Souza

DOI: $10.47094 / 978-65-88958-44-5 / 79-83$

CAPÍTULO 10

AÇÕES DO ENFERMEIRO PARA À PROMOÇÃO DA SAÚDE DA POPULAÇÃO LGBT: UMA REVISÃO BIBLIOGRÁFICA

Hemerson Felipe Fernandes abreu

Ana Paula Cunha Duarte

Linielce Portela Nina da Silva

Patrícia da Silva Pereira dos Reis 
Kelly Rose Pinho Moraes

Caroline Natielle Rocha da Silva

Anádia Nathália Matos Araújo Sousa

Antônia Josana Farias

Antônia Katia Lopes Araújo

Fabricia Da Silva Pereira Dos Reis

DOI: 10.47094/978-65-88958-44-5/84-89

CAPÍTULO 11 .090

SÍNDROME DE FOURNIER ASSOCIADA A FÍSTULA ANORRETAL: UM RELATO DE CASO

Hemerson Garcia de Oliveira Silva

Carolina Corrêa Lima

Giselle Pena de Oliveira

Henrique de Souza Rodrigues Fajardo

Alex Loze Rocha

Iago Gama Pimenta Murta

Marcos Vinícius Mendes Barroso

Robson da Silveira

Ana Luiza Soares Toledo

Tadeu Kruschewsky Midlej Neto

Lucianne Vanelle Sales Freitas

Poliana Gomes Pereira

DOI: 10.47094/978-65-88958-44-5/90-96 
CAPÍTULO 12.

RE-LEITURA DOS ESPAÇOS DE CONSTRUÇÃO DO CONHECIMENTO PARA A FORMAÇÃO INTERPROFISSIONAL DO RESIDENTE EM SAÚDE

Marília Ximenes Freitas Frota

Joana Angélica Marques Pinheiro

Maria Gyslane Vasconcelos Sobral

Glauber Gean de Vasconcelos

Renata di Karla Diniz Aires

Raquel Sampaio Florêncio

Vera Lúcia Mendes de Paula Pessoa

DOI: 10.47094/978-65-88958-44-5/97-110 


\section{CAPÍTULO 12}

\section{RE-LEITURA DOS ESPAÇOS DE CONSTRUÇÃO DO CONHECIMENTO PARA A FORMAÇÃO INTERPROFISSIONAL DO RESIDENTE EM SAÚDE}

\section{Marília Ximenes Freitas Frota ${ }^{1}$;}

Universidade Estadual do Ceará (UECE), Fortaleza, Ceará.

https://orcid.org/0000-0003-3761-0892

Joana Angélica Marques Pinheiro²;

Universidade Estadual do Ceará (UECE), Fortaleza, Ceará.

https://orcid.org/0000-0003-3092-3936

Maria Gyslane Vasconcelos Sobral ${ }^{3}$;

Universidade Estadual do Ceará (UECE), Fortaleza, Ceará.

https://orcid.org/0000-0003-1754-1009

\section{Glauber Gean de Vasconcelos ${ }^{4}$;}

Universidade Estadual do Ceará (UECE), Fortaleza, Ceará.

https://orcid.org/0000-0003-1598-4412

\section{Renata di Karla Diniz Aires;}

Universidade Estadual do Ceará (UECE), Fortaleza, Ceará.

https://orcid.org/0000-0003-4150-0549

\section{Raquel Sampaio Florêncio ${ }^{6}$;}

Universidade Estadual do Ceará (UECE), Fortaleza, Ceará.

https://orcid.org/0000-0003-3119-7187

Vera Lúcia Mendes de Paula Pessoa?.

Universidade Estadual do Ceará (UECE), Fortaleza, Ceará.

https://orcid.org/0000-0002-5441-5311 
RESUMO: Objetivo: Refletir sobre as interfaces da construção de conhecimento no âmbito dos Programas de Residência Multiprofissional em saúde, identificando aspectos que possam interferir e contribuir para a formação interprofissional do residente. Método: Trata-se de um ensaio reflexivo que emergiu durante a disciplina de Metodologia do Ensino, do Programa de Pós-graduação em Cuidados Clínicos em Enfermagem e Saúde, no ano de 2019, na Universidade do Estado do Ceará, Fortaleza- CE, Brasil. Resultados: O trabalho coletivo e interporfissional permite ir além de relações recíprocas entre condutas técnicas, interações e planejamentos envolvendo profissionais de diversas categorias num mesmo ambiente, incluindo também valores e procedimentos pautados na cooperação e comunicação, no intuito de juntos, articularem ações em prol do paciente. Entretanto, a precarização e a flexibilização das relações de trabalho no âmbito das Residências proporcionam prejuízos para a formação do residente. Conclusão: A reestruturação e re-leitura dos espaços de formação dos profissionais numa vertente mais interprofissional permite uma visão ampliada das políticas e do cuidado ao paciente. Nesse contexto evidencia-se a necessidade de construções e reconstruções no processo de formação dos residentes e de seus formadores, de maneira a sensibilizar todos os envolvidos quanto a essa nova perspectiva de conhecimento compartilhado na atuação conjunta, e assim construir estratégias para lidar com impasses existentes na assistência para beneficiar a saúde como um todo e o usuário.

PALAVRAS-CHAVE: Educação Interprofissional. Internato e Residência. Conhecimentos, Atitudes e Prática em Saúde.

\section{RE-READING OF KNOWLEDGE BUILDING SPACES FOR INTERPROFISSIONAL TRAINING OF HEALTH RESIDENTS}

ABSTRACT: Objective: To reflect about the interfaces of knowledge construction of Multiprofessional Residency Programs in health, identifying aspects that may interfere and contribute to the resident's interprofessional training. Method: Reflective essay, that emerged during the Teaching Methodology discipline, from the Postgraduate Program in Clinical Care in Nursing and Health, in 2019, at the State University of Ceará, Fortaleza-CE, Brazil. Results: Collective and inter-professional work allows us to go beyond reciprocal relationships between technical conducts, interactions and planning involving professionals from different categories in the same environment, also including values and procedures based on cooperation and communication, in order to jointly articulate actions in favor of the patient. However, the precariousness and the flexibilization of work relations in the scope of the Residences provide losses for the formation of the resident. Conclusion: The restructuring and rereading of the training spaces for professionals in a more interprofessional aspect allows an expanded view of policies and patient care. In this context, the need for constructions and reconstructions in the process of training residents and their trainers is evident, in order to sensitize everyone involved about this new perspective of shared knowledge in joint action, and thus build strategies to deal with existing impasses. assistance to benefit health as a whole and the user. 
KEY-WORDS: Interprofessional Education. Internship and Residency. Health Knowledge, Attitudes, Practice.

\section{INTRODUÇÃO}

$\mathrm{Na}$ atualidade, observa-se maior exigência em relação ao conhecimento e saber especializado dos profissionais, requisitando competências para além do científico proposto pelas universidades. Assim, com uma demanda crescente de profissionais, têm se observado modificações recorrentes nas políticas e práticas de gestão em saúde, no intuito de alcançar um perfil de trabalhador capacitado, em que já nos processos de seleção e contratação, se busca profissionais preparados quanto à técnica e prática no cuidado. Destaca-se como facilitador na preparação de tais condições, o impacto formador das residências multiprofissionais em saúde com ênfase na educação e prática interprofissional (EIP e PIP) como ferramenta imprescindível para o aprimoramento e qualificação do residente em saúde.

A formação de profissionais inseridos no Sistema Único de Saúde (SUS) tem sido fundamental para uma qualificação nas políticas públicas de saúde e da assistência aos usuários (EMERICH et al., 2017). Diante disso é proposto um novo contexto para a preparação dos recursos humanos, dentre eles podemos citar a Residência Multiprofissional em Saúde (RMPS), que preconiza uma redefinição dos conceitos e da assistência à saúde.

Os Programas de Residência Multiprofissional em Saúde utilizam como base os princípios da universalidade, integralidade, equidade, intersetorialidade, humanização do atendimento e participação social, no intuito de se aproximar das instituições de ensino e serviços de saúde, a partir da articulação entre os Ministérios da Saúde (MS) e da Educação (MEC) (BRASIL, 2007).

O objetivo da residência consiste na interiorização da Educação Permanente Interprofissional em saúde através da qualificação de profissionais, com a finalidade de contribuir para a consolidação da carreira na saúde pública e de fortalecer as Redes do SUS (GADELHA; BARRETO, 2018). A educação e a prática interprofissional (EIP E PIP) de saúde dependem de iniciativas para assegurar a melhoria da qualidade da atenção e do ensino e assim aproximar o profissional às diferentes realidades e prioridades da saúde do país.

Diante da situação foi criada em 2004 a Política Nacional de Educação Permanente em Saúde (PNEPS), como estratégia para a formação e o desenvolvimento de trabalhadores para o setor da saúde e, em especial, para o Sistema Único de Saúde (SUS) (BRASIL, 2004). Ainda nessa perspectiva, no âmbito do Ministério da Saúde e da Educação, foram criadas as Residências Multiprofissionais em Saúde (RMS), por meio da Portaria Interministerial n. 2.117/2005 (GUIMARÃES; SILVA, 2010). Em 2007, a Portaria Interministerial n. 45 reconheceu então essa modalidade de formação, como curso de especialização caracterizando-se por ensino em serviço, no nível de pós-graduação lato sensu (BRASIL, 2007). 
A residência é uma prática interprofissional, em que áreas transitam em diferentes saberes, se complementando e desenvolvendo novos conhecimentos que se propõe a atuação em uma equipe multiprofissional, como foco nas necessidades de saúde dos usuários, voltada para o futuro.

A EIP consiste na inversão da lógica tradicional da formação em saúde - cada prática profissional pensada e discutida em si, abrindo espaços para a discussão do interprofissionalismo. Uma proposta onde profissões aprendem juntas sobre o trabalho conjunto e sobre as especificidades de cada uma, na melhoria da qualidade no cuidado ao paciente. A essência da EIP em três pólos sobrepostos: preparação individual para a colaboração, estimular a colaboração entre o grupo e melhorar os serviços e a qualidade do cuidado (BARR et al., 2005).

Esse novo olhar enseja um perfil de profissionais hábeis e preparados para a tomada rápida de decisões; para assumir responsabilidades e ser resolutivo diante de situações práticas, complexas e adversas, indo além da teoria advinda de sua formação básica ou de práticas isoladas, participando ativamente nessa construção e reconstrução de saberes, baseando-se na comunicação e no diálogo.

É dessa forma que se pode fazer um diálogo com reflexões quando se coloca que existe uma necessidade da formação em saúde favorecendo a compreensão das buscas de superação de vieses presentes na preparação dos profissionais que são demonstradas apenas na compreensão de sua própria trajetória, isto é, uniprofissional (DIAS et al., 2016). Desta maneira, percebe-se a importância de um intercâmbio de saberes como forma de democratização da própria formação em saúde, como também de humanização do ensino (SANTOS; BRASILL, 2018).

A EIP preconiza o fortalecimento da equipe, articulando ações interprofissionais, representando oportunidades de formação conjunta para o desenvolvimento de aprendizagens compartilhadas, sem desvalorizar o outro e assim melhorar a qualidade da assistência ao paciente (CASANOVA; BATISTA; MORENO, 2018).

Diante disso surge a reflexão: uma re-leitura dos espaços de construção do conhecimento nos programas de RPMS, sob a ótica da educação e prática interprofissional, poderia colaborar positivamente para formação interprofissional dos residentes em saúde?

Este ensaio buscou refletir sobre as interfaces da construção de conhecimento no âmbito dos Programas de Residência Multiprofissional em saúde, identificando aspectos que possam interferir e contribuir para a formação interprofissional do residente.

\section{Uma formação ideal para residentes é possível?}

A aproximação entre a academia e os serviços de saúde são historicamente compreendidos como um processo desafiador. De fato, apenas o saber apreendido durante a formação profissional básica, ainda que estabelecido em padrões ideais de ensino e formação, em geral, não são capazes de preparar completamente o profissional para atuar nos serviços de saúde, sobretudo interprofissionalmente.

As Residências em Saúde surgem como uma ideia diferenciada de formação, carregando consigo o propósito de construir um caminho que aproxime as distâncias entre a academia e os 
serviços, através de uma articulação entre os conhecimentos teóricos e práticos.

Em se tratando de Residências Multiprofissionais em Saúde, são acrescidas novas nuances à esse modelo pois representam um diálogo entre saberes, favorecendo a integração entre diferentes competências profissionais, o que repercute positivamente na atenção e cuidado oferecido aos usuários dos serviços de saúde. Para os integrantes da RMS a EPS é encarada como algo além de uma simples ação estratégica desenvolvida no seu cotidiano, pois acreditam que ela tenha uma perspectiva maior, no cotidiano de sua atuação com o outro (SILVA et al., 2016).

A RMPS traz em sua essência uma possibilidade estratégica de transformação da organização dos serviços, do processo de formação, das ações de saúde, e das próprias práticas pedagógicas que, com o frescor trazido pelo residente, caminham para a configuração de novos processos de trabalho, articulando as instituições formadoras com os serviços assistenciais (CASANOVA; BATISTA; MORENO, 2018).

A interprofissionalidade preconizada nas RMPS's é uma busca por construir uma prática colaborativa talvez pouco ou não trabalhada durante a graduação, onde os profissionais buscam não apenas aprender a trabalhar em equipes compostas por múltiplas categorias, mas, desenvolver um processo de trabalho harmônico e construtivo, que transcenda as fronteiras do saber fragmentado.

Assim, a RMPS, pautada na formação interprofissional, pode representar a oportunidade de aprofundamento e de saberes compartilhados, construídos conjuntamente, desenvolvendo abordagens diferenciadas do processo saúde-doença, competências comuns, aprendizagem colaborativa, articulação teórica e prática para a resolução compartilhada de problemas, e o respeito mútuo entre locais de fala distintos (AGUILAR-DA-SILVA; SCAPIN; BATISTA, 2011).

Existem inúmeras vantagens atreladas à formação através de Programas de Residência. Destaca-se o fato de o residente, talvez pela renovação anual com entradas de novas turmas, trazer consigo o estímulo de construir novos saberes, e o desejo de aprender e trabalhar, revigorando, assim, os serviços. A Residência, também, tem-se mostrado mais produtiva em termos de especialização e aprimoramento de aprendizado quando comparado a outras modalidades de pós-graduação, por dispor de uma carga horária mais vasta, dentre outras características (FAJARDO; ROCHA; PASINI, 2010). Além disso, o compartilhamento de práticas a favor dos usuários são oportunidades ímpares de integração, aprendizado e fortalecimento dos princípios do SUS.

Reconhecer a RMPS como uma forma de fortalecer e qualificar o SUS é fazer valer a promoção da integralidade ao usuário dos serviços de saúde. Desde sua concepção, os objetivos das RMPS's foram qualificar a formação de profissionais de saúde e contribuir com mudanças significativas e condizentes com os preceitos do SUS nos cenários de saúde (FAJARDO; ROCHA; PASINI, 2010).

Na perspectiva de consolidação destes programas, nota-se na prática, ainda que não da maneira ideal ou esperada, uma crescente construção de espaços de articulação de saberes e diálogo entre diferentes perfis e competências profissionais, numa busca compromissada de incluir a humanização e a integralidade da assistência nas rotinas dos serviços. 
Ainda que permeada por desafios, a RMPS tem a possibilidade de transformar o "desconforto" provocado por uma experiência relativamente nova aos profissionais, antes, condicionados a práticas talvez engessadas e pouco articuladas, em um movimento de repensar o modo de se "fazer saúde".

A chegada de residentes multiprofissionais em um serviço de saúde pode provocar uma desestabilização de práticas cristalizadas, culminando na construção de novos saberes, não apenas para os residentes, mas também para os trabalhadores (FAJARDO; ROCHA; PASINI, 2010).

As RMPS têm potencialidades para provocar mudanças estruturais na forma como é concebido o processo saúde-doença e na forma como os profissionais de saúde configuram-se em suas relações de trabalho e interações com os usuários. Busca-se que, com os Programas, se alcancem efetivamente as transformações pelas quais historicamente se lutam, contribuindo para o surgimento de um terreno fértil para a consolidação do que está contido na Constituição do Sistema Único de Saúde.

\section{Fatores intervenientes na formação interprofissional do residente}

Os cursos de residências em saúde exigem um suporte de preceptores especialistas, que devem deter domínio das atividades de cunho técnico e assistencial. No entanto, temos uma deficiência de preceptores, a contratação de profissionais inabilitados à docência para repor essa falta, além de desligamentos de profissionais com maior experiência prática e melhor habilidade para promover a aquisição de conhecimento pelos residentes (NOGUEIRA; ANDRADE; SANTOS, 2019).

A precarização do trabalho assistencial e a consequente flexibilização das relações trabalhistas decorrente do modelo neoliberal, em que predominam contratos temporários e vínculos empregatícios distintos, como através de cooperativas, proporcionam prejuízos também para a formação do residente, devido à pouca experiência dos trabalhadores temporários. Acredita-se que isso se mostre como um obstáculo aos objetivos propostos no programa de residência pelo fato de que o residente, nessas circunstâncias, possa ser levado a assumir a realização de atividades que seriam privativas do profissional preceptor.

Estes fatores intervenientes no processo pedagógico da residência multiprofissional dos serviços de saúde, culminam por colocar aos gestores a necessidade inquisitória de escuta e flexibilização, com intuito de amenizar questões que envolvem o processo de trabalho e o pedagógico, as relações humanas, as mudanças constantes nas políticas e tecnologias do cuidado, além de precisar acompanhar e entender docentes e discentes como atores e, com isso, cidadãos de uma sociedade em mudança.

O gestor deve ter por norte alavancar esforços com o intuito de proporcionar condições mais favoráveis a formação, o que mostra-se primordial para o alcance dos objetivos a presença de profissionais capacitados e envolvidos com a proposta de trabalho, onde o preceptor exerça papel essencial na condução do residente, com supervisão e práticas assistenciais seguras (AGUIAR et al., 2005). 
O preceptor é ator neste processo como facilitador no processo de formação e atuação do profissional em especialização, logo responsável pelo acompanhamento do desempenho em suas atividades teórico-práticas. Assim, é necssário a realização de planejamentos cada vez mais articulados entre os programas e os serviços da instituição que recebem os residentes, bem como a valorização dos preceptores como fundamentais para que transformações duradouras aconteçam nos serviços (OLIVEIRA et al., 2017).

As deficiências pelas quais passam as instituições de saúde, podem proporcionar significativas limitações na formação do residente, advindas da observação de técnicas nem sempre ideais ou de condutas inapropriadas por parte dos próprios profissionais que, em determinadas circunstâncias, para manterem a continuidade da assistência, recorrem à improvisação, afetando de modo substancial a segurança do paciente e a qualidade do atendimento devido ao risco ao paciente com implicações éticas e legais. Em situações de tal proporção, observam-se riscos à saúde e ao bem-estar do residente, em decorrência da exposição a agentes por via biológica, devido à ausência de equipamentos de proteção individual na realização do trabalho (VARGAS; LUZ, 2010).

Como parte dessa política, deve-se considerar também a conscientização e a participação dos profissionais e residentes na aquisição e adoção de medidas de prevenção dos riscos e promoção da saúde, o que pode minimizar o absenteísmo e o adoecimento. Salienta-se que, a partir dos saberes expressos pelos sujeitos que realizam a atividade com foco na exposição a riscos ocupacionais, é relevante a participação destes, na medida em se realiza um diagnóstico do processo saúde/doença/ trabalho, pois como parte de um diálogo sinérgico com os protagonistas, obtêm-se elementos para a busca por soluções para tais demandas (FONTANA; LAUTERT, 2011).

Um contexto de formação mal estruturado pode repercutir na subjetividade do residente e acarretar desconforto e sofrimento no processo de formação especialmente em situações onde o profissional não consiga realizar um serviço de boa qualidade, criando-se um ambiente de trabalho desanimador marcado por desmotivação e insegurança; o que desencadeia estresse ocupacional com repercussões para a saúde do indivíduo, diante da insatisfação e do baixo apoio social. Havendo necessidade assim de estratégias voltadas para a valorização e cuidado dos trabalhadores, além de investimento na educação permanente, os quais podem ter papel protetor para a saúde, na medida em que proporcionam melhores condições e suporte para os profissionais atuarem de forma digna (NEGELISKII; LAUTERT, 2011).

A satisfação com o trabalho está entre os fatores reconhecidos como redutores do estresse, sendo determinante para a permanência do trabalhador no emprego, garantindo também melhor desempenho nas atividades. Frente a isso, destaca-se a relevância de se debater acerca das condições de trabalho dos residentes, com o objetivo de implementar ações que visem preparar, prevenir e minimizar situações desconfortáveis que possam surgir. Acredita-se que se devam incluir não só estratégias individuais de mudança de comportamento, mas também, e principalmente, mudanças organizacionais voltadas para proporcionar mais satisfação no ambiente de trabalho (THEME FILHA; COSTA; GUILAM, 2013). 
Existe um fenômeno de negação cultural explícita e implícita de muitos profissionais, onde se restrinja apenas à chefia do serviço ou à coordenação da residência o controle por todas as ações que envolvam o residente, incluindo gestão, ensino e assistência. A ocorrência dessa negação acerca de situações adversas é mérito muitas vezes de instituições, que geram assistência burocratizada, com viés ético ditado pela racionalização e pouco realistas (SOCIEDADE BRASILEIRA DE ANESTESIOLOGIA, 2013).

O desenvolvimento de processos avaliativos é vital para fortalecer a imagem da residência, e do profissional residente, para estabelecer uma visão de crescimento do conjunto de necessidades qualitativas indissociáveis da segurança, aprimoramento e confiança no trabalho. Alguns estudos apresentam dados a esse respeito que foram obtidos em oficinas, simpósios e levantamentos sucessivos e que apontam para fatores intervenientes na qualidade do convívio e do trabalho. A utilização de questionários e a realização de oficinas multidisciplinares diminuem os espaços de desconhecimento entre serviços, determinaram reformas relevantes para a melhoria da qualidade de vida dos residentes e aumentam a legitimidade da presença do residente em ambiente hospitalar (SOCIEDADE BRASILEIRA DE ANESTESIOLOGIA, 2013).

Refletir acerca do cotidiano do residente se mostra essencial para que se promovam ações voltadas ao seu bem estar no momento do aprendizado, minimizando a ocorrência de não conformidades que por sua vez apresentam impacto deletério sobre a qualidade da experiência, como a sobrecarga de tarefas, fadiga e ansiedade extrema, relacionamento precário com outros profissionais de saúde, pouco tempo livre para o estudo, lazer e sono restritos, alimentação inadequada, entre outras.

Faz-se necessário um olhar atento por parte da instituição formadora e do serviço que recebe o residente, com ações voltadas a inteligência emocional além dos aspectos científicos, com comportamentos e atitudes, sobretudo, preparando o jovem profissional a aprender a lidar com situações críticas e/ou pressões decorrentes da alta demanda assistencial. Todavia, vale ressaltar que de maneira geral falta equacionar problemas básicos existentes na programação da residência brasileira; no exercício de valorização de virtudes pessoais, de humanidades e de como ajudar nossos jovens na interlocução de seus problemas comportamentais e existenciais (SOCIEDADE BRASILEIRA DE ANESTESIOLOGIA, 2013).

\section{A interprofissionalidade e sua contribuição nos espaços de formação do profissional residente}

A interprofissionalidade surge exatamente num momento de necessidade de fortalecimento do sistema de saúde, no intuito de propiciar melhora na assistência ao usuário, mas também de possibilitar melhor formação profissional e de atuação, tanto por parte dos residentes como também por parte dos preceptores, em termos de melhor capacitação e ações voltadas a integralidade, resolutividade, humanização e educação continuada em saúde. 
A admissão formal da interprofissionalidade influencia nos diversos modos de trabalho. As parcerias que se reconhecem colaborativas e a colocação na cena do cotidiano, tanto pela intercomplementaridade dos saberes, como pelas práticas, exigem e favorecem um agir em equipes integradas para compor um trabalho em conjunto, articulando atividades de grupo num processo participativo e efetivo na sua educação permanente, envolvendo também o problematizar das relações de poder e a transformação de conflitos e disputas, utilizando para tal, estratégias didático-pedagógicas de confronto de idéias e negociação (CECCIM, 2018).

Observa-se então que a contribuição interprofissional na produção do cuidado é que permite o aprimoramento na formação desse profissional-residente num cenário real de atuação em conjunto, mediante encontro de diferentes subjetividades nos campos de saberes e práticas integradas, complementando-se, favorecendo uma noção de totalidade ao profissional (REIS; DAVID, 2010).

Assim, acredita-se que a atuação de residentes, de diversas categorias profissionais, junto a uma equipe de saúde interdisciplinar, propicie melhor assistência, em termos de qualidade e quantidade de atendimentos aos usuários, na medida em que prestam o cuidado com um olhar ampliado, com apoio de diferentes especialidades e troca significativa de conhecimentos a partir do debate acerca de cada paciente, seu histórico e planos terapêuticos elaborados (PEDUZZI, 2001).

Esse trabalho coletivo e interporfissional permite o ir além de relações recíprocas entre condutas técnicas, interações e planejamentos envolvendo profissionais de diversas categorias num mesmo ambiente, incluindo também valores e procedimentos pautados na cooperação e comunicação, no intuito de juntos, articularem ações em prol do paciente. Diante de realidades assim, destaca-se mais uma vez a potencialidade relacionada à uma boa formação de residentes para que possam atuar de maneira eficiente, mostrando-se presença impactante para o fortalecimento de atuação nas Redes de Atenção à Saúde do SUS.

Podemos dizer que a contribuição da atuação do residente não se restringe apenas ao usuário pois na realidade do serviço, o preceptor também tem seu modo de agir impactado e estimulado por essa prática assistencial diária conjunta, numa ação interprofissional, desencadeando assim novos olhares, parcerias, renovações e mudanças em relação às técnicas e atividades que habitualmente eram utilizadas naquele âmbito de cuidado, promovendo ações voltadas aquela população acompanhada.

Uma educação marcada pela interporfissionalidade promove de forma efetiva um aprendizado compartilhado de competências com estudantes e profissionais de outras áreas. Numa visão mais ampliada, não se trata de um aprendizado que ocorrer isoladamente nas salas de aula, mas sim de um aprendizado interativo com colegas de outras profissões nos cenários de prática. $\mathrm{O}$ aprendizado se daria na medida em que estudantes convivem em experiências reais com profissionais e estudantes de outras áreas (TOASSI, 2017).

Uma formação de profissionais em campos de práticas tendo como base os princípios da integralidade e à interprofissionalidade pode ser encarado como algo inovador e desafiador exigindo um processo de construções e reconstruções contínuas das instituições formadoras e dos preceptores. Diante disso pode-se afirmar que para a profissionalização, capacitação e sensibilização destes, faz- 
se necessária a instauração de programas contínuos de desenvolvimento docente para os preceptores que fomentem a inovação e transformação do processo de formação em saúde (PARO; PINHEIRO, 2018).

Acredita-se que a parceria entre ensino, serviço e comunidade se mostre benéfica e fortaleça a proposta inicial da residência, como mencionada anteriormente, como modelo de formação com melhor impacto na saúde e com formas inovadoras de enfrentar a prática assistencial (KUABARA; TONHOM; MARIN, 2016). Para isso, é pertinente reforçar que universidades e instituições de ensino superior sigam o modelo do SUS e busquem estabelecer diálogo entre residentes, preceptores e serviços, repensando também seus projetos de formação, não somente no que diz respeito a transformação de concepções teóricas e práticas, mas, sobretudo, nas relações de poder dentro das universidades, dos serviços de saúde e do território de atuação.

\section{CONSIDERAÇÕES FINAIS}

Diante dessa reflexão podemos evidenciar que as RMPS representam um novo espaço de aprendizado e formação, permitindo a capacitação de residentes multiprofissionais na área da saúde, proporcionando uma visão integral e interdisciplinar do seu espaço de atuação.

Ainda é possível se constatar na graduação uma formação unidisciplinar, mesmo que na realidade atual tanto se fale em multi e interdisciplinaridade, o que muitas vezes pode levar os alunos a um pensamento individualista e pouco generalista. Tal condição pode sofrer transformação no decorrer de sua preparação baseada na interprofissionalidade em RMPS, porém essa tal renovação só acontecerá aos que tiverem a oportunidade de vivenciar essa realidade. A possibilidade de aprender a ter um olhar e um pensamento ampliado permitem o desenvolvimento de políticas públicas direcionadas para um cuidado integral dos usuários do Sistema Único de Saúde.

Apesar de ser um espaço rico em conhecimento podemos observar vários obstáculos enfrentados por esses profissionais na sua formação, como uma condução realizada por preceptores pouco capacitados e despreparados, falta de condições de trabalho em relação a espaço físico, materiais e insumos ideiais para o desenvolvimento de uma prática adequada, deparando-se com uma real condição de precarização do trabalho e com a falta de investimento público em relação à sustentação das ações em saúde, inclusive muitas vezes supridas pelo residente, como mão de obra na assistência.

Em contrapartida são proporcionados momentos de aprendizado riquíssimos na formação dos residentes, entre eles podemos citar: os momentos de interações interdisciplinares, planejamento de atividades pela equipe, encontros com profissionais e usuários na visão interdisciplinar e espaços em que residentes/preceptores dialogam sobre o desenvolvimento do seu processo de formação. Assim, a RMPS, embasada numa formação interprofissional, promove oportunidade de compartilhar saberes no processo saúde-doença numa atuação colaborativa entre os profissionais, modificando o padrão de assistência categorizada e já ultrapassada. 
Conforme exposto, a reestruturação e re-leitura dos espaços de formação dos profissionais numa vertente mais interprofissional permite uma visão ampliada das políticas e da vivencia do cuidado ao paciente. Pode-se dizer que uma formação de RMPS com diretrizes incluindo múltiplos saberes deva ser utilizada como uma estratégia de propagação do conhecimento teórico e prático, proporcionando assim, o desenvolvimento inovação de ideologias transformadoras no SUS, bem como mudanças na atuação e atitude dos profissionais frente a um cuidado diferenciado e integral ao usuário proporcionando um atendimento de melhor qualidade.

Nesse contexto evidencia-se a necessidade de construções e reconstruções no processo de formação dos residentes e de seus formadores, de maneira a sensibilizar todos os envolvidos quanto a essa nova perspectiva de conhecimento compartilhado na atuação conjunta, e assim construir estratégias para lidar com impasses existentes na assistência, beneficiando o usuário.

Buscamos nesse estudo proporcionar uma ampliação quanto à discussão dos novos espaços de formação dos profissionais/residentes, destacando a vertente da interprofissionalidade nesse cenário, mostrando expectativas, dificuldades, perspectivas e contribuições. Vale ressaltar que ainda existe uma importante lacuna na produção cientifica acerca das RMPS embasadas na interprofissionalidade tanto em sua formação quanto na atuação, no entanto podemos evidenciar significativo desenvolvimento de produções cientificas envolvendo as RMPS com temáticas voltadas a atenção básica em saúde, o que também nos faz refletir sobre a necessidade de publicações relacionadas a atenção secundária e terciária em saúde.

\section{DECLARAÇÃO DE INTERESSES}

Nós, autores deste artigo, declaramos que não possuímos conflitos de interesses de ordem financeira, comercial, político, acadêmico e pessoal.

\section{REFERÊNCIAS}

AGUIAR, B. G. C. et al. Guia de orientações para o enfermeiro residente: Curso de Pós-Graduação (Especialização), sob a Forma de Treinamento em Serviço (Residência) para Enfermeiros (Residência em Enfermagem). Brasília, DF: Ministério da Saúde, 2005. Disponível em: http://bvsms.saude.gov. br/bvs/publicacoes/guia_orientacoes_enfermeiros_residentes.pdf. Acesso em: 11 jan 2021.

AGUILAR-DA-SILVA, S. R. H.; SCAPIN, L. T.; BATISTA, N. A. Avaliação da formação interprofissional no ensino superior em saúde: aspectos da colaboração e do trabalho em equipe. Avaliação Rev da Avaliação da Educ Super, v. 16, n. 1, p. 165-84, 2011. Disponível em: http://dx.doi. org/10.1590/S1414-40772011000100009. Acesso em: 14 jan 2021.

BARR, H. et al. Effective Interprofessional Education [Internet]. Oxford, UK: Blackwell Publishing Ltd, 2005.

BRASIL. Ministério da Educação. Portaria interministerial no 45, de 12 de janeiro de 2007. Institui 
a comissão nacional de residência multiprofissional em saúde. Ministério da Educação, DF: Diário Oficial da União [online], 2007. Disponível em: http://portal.mec.gov.br/sesu/arquivos/pdf/residencia/ portaria_45_2007.pdf. Acesso em: 16 jan 2021.

BRASIL. Ministério da Saúde. Portaria nº 198, de 13 de fevereiro de 2004. Institui a Política Nacional de Educação Permanente em Saúde como estratégia do Sistema Único de Saúde para a formação e o desenvolvimento de trabalhadores para o setor e dá outras providências. Ministério da Saúde, DF: Diário Oficial da União, 2004. Disponível em: https://www.nescon.medicina.ufmg.br/biblioteca/ imagem/1832.pdf.Acesso em: 13 jan 2021.

CASANOVA, I. A.; BATISTA, N. A.; MORENO, L. R. A Educação Interprofissional e a prática compartilhada em programas de residência multiprofissional em Saúde. Interface - Comun Saúde, Educ, v. 22, n. 1, p. 1325-37, 2018. Disponível em: http://dx.doi.org/10.1590/1807-57622017.0186. Acesso em: 15 jan 2021.

CECCIM, R. B. Conexões e fronteiras da interprofissionalidade: forma e formação. Interface Comun Saúde, Educ, v. 22, n. 2, p. 1739-49, 2018. Disponível em: http://dx.doi.org/10.1590/180757622018.0477. Acesso em: 13 jan 2021.

DIAS, I. et al. Educação interprofissional e formação em saúde: pontes e diálogos. Porto Alegre: Rede Unida, 2016.

EMERICH, B. F. et al. Manual do programa de residência multiprofissional em saúde mental. Campinas, SP: Unicamp, 2017. 29 p. Disponível em: https://www.fcm.unicamp.br/fcm/sites/default/ files/2017/page/manual_do_programa_de_residencia_multiprofissional_em_saude_mental_-_ digital.pdf. Acesso em: 12 jan 2021.

FAJARDO, A. P.; ROCHA, C. M. F.; PASINI, V. L. Residências em saúde: fazeres \& saberes na formação em saúde. Porto Alegre: Hospital Nossa Senhora da Conceição, 2010. Disponível em: http://www.sbrafh.org.br/site/public/temp/4f7baaa8ca532.pdf. Acesso em: 14 jan 2021.

FONTANA, R. T.; LAUTERT, L. The situation of nursing work and occupational risks from an ergological perspective. Rev Lat Am Enfermagem, v. 21, n. 6, p. 1306-13, 2013. Disponível em: http://dx.doi.org/10.1590/0104-1169.3105.2368. Acesso em: 14 jan 2021.

GADELHA, A. K. S.; BARRETO, I. C. H. C. Residência integrada em Saúde: percepção dos atores da ênfase em Saúde da Família e Comunidade. Interface - Comun Saúde, Educ, v. 22, n. 1, p. 133951, 2018. Disponível em: http://dx.doi.org/10.1590/1807-57622017.0183. Acesso em: 18 jan 2021.

GUIMARÃES, D. A.; SILVA, E. S. Formação em ciências da saúde: diálogos em saúde coletiva e a educação para a cidadania. Cien Saude Colet, v. 15, n. 5, p. 2551-62, 2010. Disponível em: http:// dx.doi.org/10.1590/S1413-81232010000500029. Acesso em: 18 jan 2021.

KUABARA, C. T. M.; TONHOM, S. F. R.; MARIN, M. J. S. Integração ensino-serviço na perspectiva dos profissio- nais da rede primária de saúde. Revista fórum identidade, v. 22, n. 22, p. 173-192, 2016. Disponível em: https://www.seer.ufs.br/index.php/forumidentidades/article/view/6214/5178. Acesso 
em: 16 jan 2021.

NEGELISKII, C.; LAUTERT, L. Occupational stress and work capacity of nurses of a hospital group. Rev Lat Am Enfermagem, v. 19, n. 3, p. 606-13, 2011. Disponível em: http://dx.doi.org/10.1590/ S0104-11692011000300021. Acesso em: 12 jan 2021.

NOGUEIRA, E. S.; ANDRADE, E. G. S.; SANTOS, W. L. Assistência de enfermagem no transplante de córnea. Rev Inic Cient Ext., v. 2, n. 2, p. 89-95, 2019. Disponível em: https://revistasfacesa. senaaires.com.br/index.php/iniciacao-cientifica/article/view/151. Acesso em: 14 jan 2021.

OLIVEIRA, E. B. et al. Factors involved in the training of resident nurses: view of alumni from a residency program. REME Rev Min Enferm, v. 21, 2017. Disponível em: http://dx.doi. org/10.5935/1415-2762.20170074. Acesso em: 17 jan 2021.

PARO, C. A.; PINHEIRO, R. Interprofissionalidade na graduação em Saúde Coletiva: olhares a partir dos cenários diversificados de aprendizagem. Interface - Comun Saúde, Educ, v. 22, n. 2, p. 1577-88, 2018. Disponível em: http://dx.doi.org/10.1590/1807-57622017.0838. Acesso em: 12 jan 2021.

PEDUZZI, M. Equipe multiprofissional de saúde: conceito e tipologia. RevSaude Publica, v. 35, n. 1, p. 103-9, 2001. Disponível em: http://www.scielo.br/pdf/rsp/v35n1/4144.pdf. Acesso em: 16 jan 2021.

REIS, V. M.; DAVID, H. M. S. L. O fluxograma analisador nos estudos sobre o processo de trabalho em saúde: uma revisão crítica. Rev APS, v. 13, n. 1, p. 118-25, 2010. Disponível em: https://periodicos. ufjf.br/index.php/aps/article/view/14347. Acesso em: 12 jan 2021.

SANTOS, L. V.; BRASIL, M. L. Educação e Saúde na Perspectiva Interprofissional: Programa de Educação pelo Trabalho para a Saúde - Redes de Atenção à Saúde - PET-RAS. Cien Saude Colet, v. 23, n. 7, p. 2453-4, 2018. Disponível em: http://dx.doi.org/10.1590/1413-81232018237.29862016. Acesso em: 15 jan 2021.

SILVA, C. T. et al. Residência multiprofissional como espaço intercessor para a educação permanente em saúde. Texto Context - Enferm, v. 25, n. 1, 2016. Disponível em: http://dx.doi.org/10.1590/01040707201600002760014 . Acesso em: 14 jan 2021.

SOCIEDADE BRASILEIRA DE ANESTESIOLOGIA. Residentes de anestesiologia: importância do bem-estar ocupacional. Bem-estar ocupacional em anestesiologia. Brasília, DF: Conselho Federal de Medicina, 2013. Disponível em: https://portal.cfm.org.br/images/stories/biblioteca/bem\%20 estar\%20em\%20anestesiologia.pdf. Acesso em: 12 jan 2021.

VARGAS, M. A. O; LUZ, A. M. H. Práticas seguras do/no cuidado de enfermagem no contexto hospitalar: é preciso pensar sobre isso e aquilo. Enferm. Foco., v. 1, n. 1, p. 23-7, 2010. Disponível em: http://revista.cofen.gov.br/index.php/enfermagem/article/viewFile/5/6. Acesso em: 14 jan 2021.

THEME FILHA, M. M.; COSTA, M. A. S.; GUILAM, M. C. R. Estresse ocupacional e autoavaliação de saúde entre profissionais de enfermagem. Rev. Latino-Am. Enfermagem, v. 21, n. 2, p. 1-9, 2013. 
Disponível em: http://www.scielo.br/pdf/rlae/v21n2/pt_0104-1169-rlae-21-02-0475.pdf. Acesso em: 15 jan 2021.

TOASSI, R. F. C. Interprofissionalidade e formação na saúde: onde estamos?. Porto Alegre: Rede Unida, 2017. Disponível em: http://historico.redeunida.org.br/editora/biblioteca-digital/serievivencias-em-educacao-na-saude/vol-06-interprofissionalidade-e-formacao-na-saude-pdf. Acesso em: 16 jan 2021. 


\section{Índice Remissivo}

$\mathbf{A}$

Abordagem médica 91, 93

Acadêmicos de enfermagem 28, 30, 32

Acessibilidade 34, 71, 73, 77

Ações de enfermagem 85, 86

Adaptação da equipe 14, 17

Ansiedade 23, 37, 39, 40, 41, 42, 44, 47, 104

Antibióticos 91, 93, 94

Antibioticoterapia 91, 93, 94, 95

Aplicativo 'socrative' 71, 73

Aprendizagem 66, 71

Atenção ao usuário da saúde mental 35, 41

Atenção primária à saúde $15,42,54,60,85,86$

Atendimento 14, 16, 17, 20, 21, 24, 25, 35, 37, 38, 44, 45, 46, 47, 48, 49, 54, 55, 56, 57, 58, 59, 66, 67, 87, 88, 95, 99, 103,107

Atitudes e prática em saúde 98

Atividade física na saúde mental 35, 37

Atividades terapêuticas 35

C

Call-center covid-19 52, 55

Campanha de vacinação $28,29,30,32,33$

Centro de atendimento psicossocial (caps) 35

Colostomia 91, 94, 95

Conhecimentos 98

Coronavírus 13, 14, 17, 50, 55, 57, 60

Covid-19 13, 14, 15, 16, 17, 18, 29, 30, 48

Cuidados clínicos 98

Cuidados paliativos 64, 66

Cuidados primários à saúde 14

D

Desparamentação 64, 66

E

Edema 91, 92, 93

Educação em saúde 43, 45, 46, 48, 49, 50, 87

Educação interprofissional 98, 108

Emergência cirúrgica 91, 93

Empoderamento da equipe de enfermagem 28

Enfermeiro 85, 86

Engajamento de professores e estudantes 64

Equipe multiprofissional 14 
Equipes de saúde $65,80,81$

Estratégia de comunicação virtual 52, 59

Estratégia de saúde da família 6, 14, 15, 16, 55

Estresse 39, 40, 44, 45, 47, 103

Ética $15,18,28,29,33,34,55$

Exercício físico 35, 36, 37, 39, 40, 41, 48

Experiência e vivências de residentes de saúde da família 52

F

Fila de prioridade 28

Formação interprofissional do residente 98, 100, 102

Formação profissional 64, 100, 104

G

Gestão das tecnologias de informação e comunicação 52

Gestão do cuidado à saúde 52, 54

Grangrena de fournier 91, 92

H

Hiperemia 91, 92, 93

Hospitais 20, 21, 25, 26

I

Impactos da pandemia 20

Implicações bioéticas 28

Imunização 28, 29, 30, 31, 32, 33

Internato e residência 98

Intervenção multiprofissional em uma usf 13,15

Isolamento social $16,43,45,58,66$

L

Linha de frente $16,20,22,28,29,30,32$

M

Manejo da vacina 28, 30

Médicos 20, 22, 23, 24, 25, 26, 57, 58, 66, 67

Médicos residentes 20

Metodologias ativas 71

Microrganismos aeróbios e anaeróbios 91

\section{O}

O mundo acadêmico e a realidade prática 20, 23

Organização mundial da saúde 14, 20, 21, 53 
Pandemia $6,13,14,15,16,17,20,21,22,23,24,25,26,29,30,32,34,43,45,50,52,53,55,59,60,64,65,66,68$, 69,77

Paramentação 64, 66

Pessoas com comorbidades 44

Pessoas lgbt 85,86

Política nacional de saúde integral à população lgbt (pnsiplgbt) 85,86

Princípios bioéticos 28, 31

Princípios éticos e bioéticos 28, 30, 33

Procedimento cirúrgico 91, 93

Processo infeccioso 91, 92

Processo saúde-doença 80, 82, 101, 102, 106

Processos cirúrgicos de desbridamento 91

Profissionais de educação física 35,37

Profissionais de saúde 6, 13, 15, 16, 17, 23, 28, 31, 45, 46, 64, 65, 66, 67, 68, 87, 88, 101, 102, 104

Profissionais residentes 52

Programa de residência $13,15,16,18,20,23,45,54,82$

Programa de residência multiprofissional em saúde da família $13,15,16,18,54,82$

Programas de especialização 20,22

Promoção da saúde 35, 40, 41, 45, 46, 47, 50, 80, 81, 83, 85, 86, 87, 103

Q

Qualidade de vida $35,37,40,41,42,45,47,48,49,95,104$

$\mathbf{R}$

Recuperação de saúde 14

Rede de atenção à saúde (ras) 52

Residência em saúde 20,23, 80, 83

Residência médica 6, 20, 22, 23, 24, 25, 26

Residência multiprofissional em saúde mental e preceptora 35

Residências em saúde 52, 58, 76, 102

Residentes $6,13,15,16,17,20,22,23,24,25,26,38,43,46,52,54,55,56,58,59,60,66,76,98,100,102,103,104$, $105,106,107$

Residentes de educação física 43, 46

Residentes multiprofissionais 25, 26, 52, 55, 102, 106

$\mathbf{S}$

Sala de espera 44, 46, 49, 50

Sars-cov-2 6, 13, 14, 20, 21, 29, 32, 34, 53, 54, 69

Saúde coletiva 28, 30, 33, 41, 43, 45, 46, 50, 75, 109

Saúde da família 13, 15, 54, 80, 81, 83, 84, 89, 108

Saúde mental 35

Serviço de enfrentamento à covid-19 20, 23

Serviço de teleatendimento $52,54,55,56$

Serviços básicos de saúde 85,88 
Síndrome de fournier 91, 93, 95, 96

Sistema de saúde 13, 23, 29, 44, 49, 52, 54, 83, 104

Superação das dificuldades 71

Surtos epidemiológicos 52, 59

$\mathbf{T}$

Tecido desvitalizado 91, 93

Tecido necrótico 91, 93

Tecnologia da informação 64

Tecnologia educacional 71

Tecnologias de informação e comunicação (tic) 52

Telessaúde 64, 65, 69

Territorialização 80,82

Territorialização em saúde 80,82

Trabalho coletivo e interporfissional 98, 105

Trabalho multiprofissional 14, 17

Treinamento em serviço 52, 107

Troca de experiências 14, 17

\section{$\mathbf{U}$}

Unidade básica de saúde 43, 46, 49

Unidades de terapia intensiva 20, 21

V

Vacinação dos profissionais 28 
editoraomnisscientia@gmail.com M https://editoraomnisscientia.com.br/ @editora_omnis_scientia (0) https://www.facebook.com/omnis.scientia.9 § +55 (87) 9656-3565@ 


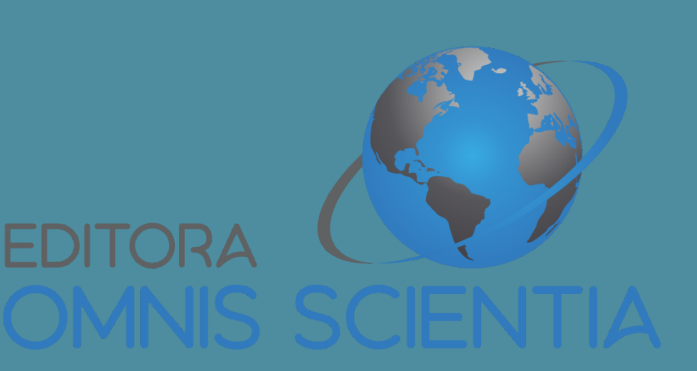

\section{editoraomnisscientia@gmail.com $\square$ https://editoraomnisscientia.com.br/ \\ @editora_omnis_scientia (0) \\ https://www.facebook.com/omnis.scientia.9 $f$ \\ +55 (87) 9656-3565@}

